\title{
Introduction of less invasive surfactant administration (LISA), impact on diagnostic and therapeutic procedures in early life: a historical cohort study
}

\author{
I. A. L. Bugter ${ }^{1}$, L. C. E. Janssen ${ }^{1}$, J. Dieleman², B. W. Kramer ${ }^{3}$, P. Andriessen ${ }^{1}$ and H. J. Niemarkt ${ }^{1 *}$ (D)
}

\begin{abstract}
Background: In preterm infants with Respiratory Distress Syndrome (RDS), Less Invasive Surfactant Administration (LISA) has been established to reduce the need of mechanical ventilation and might improve survival rates without bronchopulmonary dysplasia. The aim of this study was to investigate whether NICU care has changed after introduction of less invasive surfactant administration (LISA), with regard to diagnostic and therapeutic procedures in the first week of life.
\end{abstract}

Methods: Infants with gestational age < 32 weeks who received surfactant by LISA (June 2014 - December 2017, $n=169$ ) were retrospectively compared to infants who received surfactant after intubation (January 2012 - May $2014, n=155)$. Local protocols on indication for surfactant, early onset sepsis, blood transfusions and enteral feeding did not change between both study periods. Besides, as secondary outcome complications of prematurity were compared. Data was collected from electronic patient files and compared by univariate analysis through Students T-test, Mann Whitney-U test, Pearson Chi-Square test or Linear by Linear Association.

Results: All baseline characteristics of both groups were comparable. Compared to controls, LISA patients received a higher total surfactant dose (208 vs.160 mg/kg; $p<0.001)$, required redosing more frequently (32.5\% vs. 21.3\%; $p=0.023)$, but needed less mechanical ventilation (35.5\% vs. 76.8\%; $p<0.001)$. After LISA, infants underwent fewer X-rays (1.0 vs. 3.0, $p<0.001$ ), blood gas examinations (3.0 vs. 5.0, $p<0.001$ ), less inotropic drugs (9.5\% vs. 18.1\%; $p=$ $0.024)$, blood transfusions ( $24.9 \%$ vs. $41.9 \%, p=0.003$ ) and had shorter duration of antibiotic therapy for suspected early onset sepsis ( 3.0 vs. 5.0 days, $p<0.001)$. Moreover, enteral feeding was advanced faster ( $120 \mathrm{vs.} 100 \mathrm{~mL} / \mathrm{kg} / \mathrm{d}$, $p=0.048)$ at day seven. There were no differences in complications of prematurity.

Conclusion: The introduction of LISA is associated with significantly fewer diagnostic and therapeutic procedures in the first week of life, which emphasizes the beneficial effects of LISA.

Keywords: Preterm, Respiratory distress syndrome (RDS), Less invasive surfactant administration (LISA), Minimally invasive surfactant therapy (MIST), Surfactant, Avoidance of mechanical ventilation

\footnotetext{
* Correspondence: Hendrik.niemarkt@mmc.nl

'Department of Neonatology, Máxima Medical Centre, De Run 4600, 5504 DB Veldhoven, The Netherlands

Full list of author information is available at the end of the article
}

(c) The Author(s). 2020 Open Access This article is licensed under a Creative Commons Attribution 4.0 International License, which permits use, sharing, adaptation, distribution and reproduction in any medium or format, as long as you give appropriate credit to the original author(s) and the source, provide a link to the Creative Commons licence, and indicate if changes were made. The images or other third party material in this article are included in the article's Creative Commons licence, unless indicated otherwise in a credit line to the material. If material is not included in the article's Creative Commons licence and your intended use is not permitted by statutory regulation or exceeds the permitted use, you will need to obtain permission directly from the copyright holder. To view a copy of this licence, visit http://creativecommons.org/licenses/by/4.0/ The Creative Commons Public Domain Dedication waiver (http://creativecommons.org/publicdomain/zero/1.0/) applies to the data made available in this article, unless otherwise stated in a credit line to the data. 


\section{Background}

Non-invasive strategies in neonatal care of preterm infants are becoming increasingly important. However, a significant proportion of preterm infants with respiratory distress syndrome (RDS) fails non-invasive respiratory support alone and need exogenous surfactant (SF) replacement therapy [1]. In order to avoid intubation and mechanical ventilation, alternative approaches are developed. The most applied method of those is the INtubate-SURfactant-Extubate (INSURE) technique. However, with this technique a large proportion of infants still failed to be weaned of the ventilator immediately after surfactant treatment [2]. Other methods are still being studied, for example the INtubate-RECruiteSURfactant-Extubate (IN-REC-SUR-E) technique, SF via laryngeal mask or nebulised SF $[3,4]$. A new recently investigated technique is called less invasive surfactant administration (LISA), in which SF is administered via a thin catheter to spontaneously breathing infants on nasal continuous positive airway pressure (CPAP) [5]. Recent studies show that LISA might be associated with higher survival rates without bronchopulmonary dysplasia (BPD) and might lead to fewer other complications of preterm birth, such as severe IVH, when compared to endotracheal SF administration after intubation [6-9].

However, when we introduced LISA to our level III NICU, we observed that LISA did not only affect the need of mechanical ventilation. It also changed the way we care for these infants in early life. This includes diagnostic and therapeutic procedures, which are potentially stressful or harmful for the infant, which is in line with our focus on family centred care. In our single room maternity NICU, mother-child bonding and the avoidance of stressful events are highly valuated.

We thus hypothesized that LISA, by preventing intubation and mechanical ventilation, is associated with a reduction in diagnostic and therapeutic procedures in early life. Therefore, the aim of our study was to quantify our experience and investigate if and how care has changed since the implementation of LISA, regarding diagnostic procedures and treatment in the first week of life. As a secondary outcome, complications of prematurity were compared. It is necessary and relevant to obtain objective and quantitative insight in those potential differences in practice, since they may lead to a change in the stress burden for the infant and in healthcare costs.

\section{Methods}

A single-centre, historical cohort study was conducted in a level III NICU in The Netherlands. This study was approved by the local ethical board. Data was retrospectively extracted from electronic patient files and subsequently anonymised for analysis.

\section{Study population and data collection}

All preterm infants born between $24+0$ and $31+6$ weeks of gestation, who received SF therapy for RDS between January 2012 and December 2017, were eligible. The study population was divided into two groups as shown in Fig. 1. The control group consisted of patients who received SF after endotracheal intubation followed by mechanical ventilation (January 2012-May 2014). The intervention group consisted of patients receiving SF via LISA (June 2014 - December 2017). All patients in both study groups received CPAP $6 \mathrm{~cm} \mathrm{H}_{2} \mathrm{O}$ support directly after birth with a $\mathrm{T}$-piece resuscitator in the delivery room. Infants were only directly intubated in the delivery room in case of severe persistent apnoea. Infants in the control group who showed insufficient respiratory drive and therefore would not have qualified for LISA during the intervention period, were excluded from analysis. Patients were also excluded if they were out born and intubated before transportation to our NICU centre or if structural congenital anomalies were present. Demographic data, Apgar scores, umbilical-pH, $\mathrm{FiO}_{2}$ before surfactant (as measure of RDS severity) were collected. Local protocols on indication for surfactant, Early Onset Sepsis (EOS), blood transfusions and enteral feeding did not change between both study periods.

\section{SF treatment}

In both study periods (control and LISA), the aim was to treat with early rescue SF therapy conform the European Consensus Guidelines on the Management of Respiratory Distress Syndrome [10]. All patients received SF if they were on nasal CPAP of $6 \mathrm{~cm} \mathrm{H}_{2} \mathrm{O}$ and their fraction of inspired oxygen $\left(\mathrm{FiO}_{2}\right)$ was above 0.30 . The maximum time window for SF replacement treatment was $72 \mathrm{~h}$ after birth. Patients were treated with $100-200 \mathrm{mg} / \mathrm{kg}$ Poractant alfa (Chiesi Pharmaceuticals, Parma, Italy).

Patients in LISA group were treated according to the Dutch LISA guideline. Infants received atropine (5 micrograms $/ \mathrm{kg}$ ) and a loading dose of intravenous caffeine $(20 \mathrm{mg} / \mathrm{kg}$ ) before LISA. No sedative medication was used prior to the procedure. A 4-5 French umbilical catheter or LISAcath ${ }^{\oplus}$ (Chiesi Pharmaceuticals, Parma, Italy) was placed below the vocal cords under direct laryngoscopy, with or without the use of Magill forceps. The laryngoscope was removed, and SF was subsequently administered in 2 to $3 \mathrm{~min}$. During the procedure, nasal CPAP therapy at $6-8 \mathrm{~cm} \mathrm{H}_{2} \mathrm{O}$ was continued.

The patients in the control group were intubated after administration of atropine, fentanyl and when necessary rocuronium. Infants were extubated as soon as they met the predefined extubation criteria. Those include a sufficient respiratory drive, low positive inspiratory pressure on volume targeted ventilation $\left(<18 \mathrm{~cm} \mathrm{H}_{2} \mathrm{O}\right.$, with tidal volumes of $4-5 \mathrm{ml} / \mathrm{kg}$ ) and low $\mathrm{FiO}_{2}(<0.30)$. No medication was administered to antagonize the relatively short-acting sedatives that were used. 


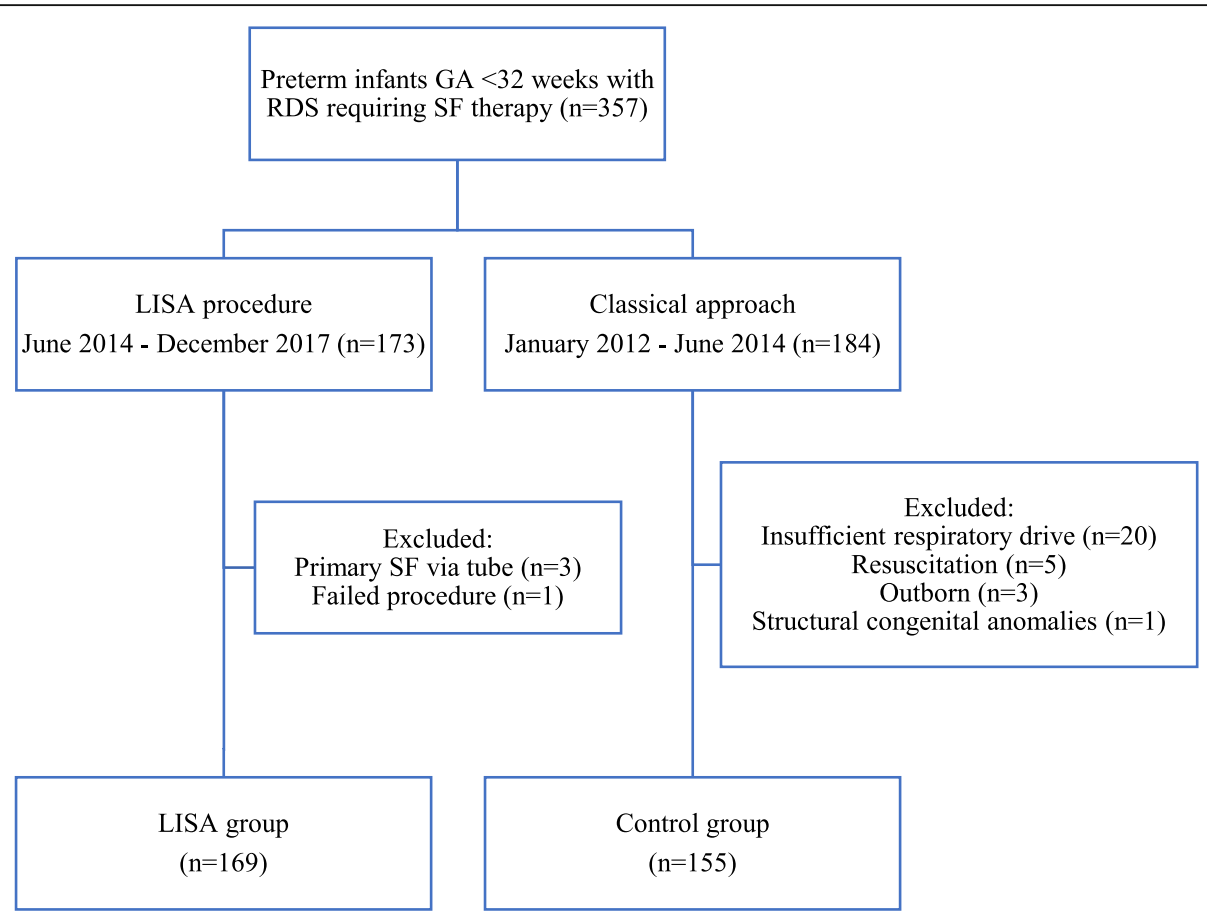

Fig. 1 Flowchart summarising the patient selection procedure

\section{Outcome measurements}

Outcome variables were divided into two different groups: diagnostic procedures and treatment in the first week of life. As secondary outcomes, complications of prematurity were compared.

Diagnostic procedures were related to the number of $\mathrm{X}$-rays, blood sample analyses and blood cultures for suspected EOS.

Outcomes regarding treatment included details on SF treatment, respiratory support, drug prescriptions, blood transfusions, enteral feeding, need for central catheters and phototherapy. SF therapy was specified with the total used dose of Poractant alfa and repeated procedures via LISA or endotracheal tube. The need for mechanical ventilation for at least $12 \mathrm{~h}$ and the absolute days on mechanical ventilation were reported. Information on the use of morphine, midazolam, inotropes, insulin, hydrocortisone and antibiotics was collected. Patent Ductus Arteriosus (PDA) treatment course included the number of courses of ibuprofen or indomethacin during NICU admission. The number of patients who underwent surgical ligation of a PDA was reported as well. Erythrocyte and thrombocyte transfusions reflect on the number of given transfusions in the first week of life. Enteral feeding was recorded as the amount of enteral nutrition given on day five and day seven in millilitres per kilogram per day.

Relevant complications of prematurity, which were reliable to find retrospectively, were supplementary oxygen on day 28 in survivors, surgical ligation of PDA, abdominal surgery on account of necrotizing enterocolitis (NEC) or spontaneous intestinal perforation (SIP), intraventricular haemorrhage (IVH) grade 3-4, pneumothorax after SF treatment, laser surgery because of retinopathy of prematurity (ROP) and mortality during NICU admission.

\section{Statistical analysis}

The statistical analysis was performed using SPSS V.22. Continuous variables were compared by Students T-test or Mann Whitney-U test, depending on distribution. Outcomes were presented as mean with standard deviation (SD) when normally distributed and as median with interquartile range (IQR) when skewed. Nominal and ordinal variables were compared by Pearson Chi-Square test and Linear by Linear Association, respectively. Considering nominal variables, when the expected count of more than $20 \%$ of the cells was fewer than 5, Fisher's Exact test was used. Those outcomes were presented as frequencies with percentages. Statistical significance was accepted at a twosided $p$-value of fewer than 0.05 .

\section{Results}

\section{Study population and baseline characteristics}

In the study cohort, $n=169$ infants received exogenous SF therapy via LISA between June 2014 and December 2017. Control group consisted of $n=155$ infants, who received SF therapy via endotracheal tube from January 2012 till June 2014. Baseline characteristics were compared and are shown in Table 1. 
Table 1 Baseline characteristics of the study population

\begin{tabular}{|c|c|c|c|}
\hline & LISA $(\boldsymbol{n}=169)$ & Control $(\boldsymbol{n}=155)$ & $\boldsymbol{p}$-value \\
\hline GA (weeks + days), mean (SD in days) & $28+0(14)$ & $28+1(14)$ & 0.52 \\
\hline GA categories & & & 0.26 \\
\hline $24-25+6$ weeks & $29(17.2 \%)$ & $21(13.5 \%)$ & \\
\hline $26-27+6$ weeks & $58(34.3 \%)$ & $50(32.3 \%)$ & \\
\hline $28-31+6$ weeks & $82(48.5 \%)$ & $84(54.2 \%)$ & \\
\hline Birth weight (grams), mean (SD) & $1092(341)$ & $1130(350)$ & 0.33 \\
\hline Birth weight categories & & & 0.44 \\
\hline$<750 \mathrm{~g}$ & $29(17.2 \%)$ & $22(14.2 \%)$ & \\
\hline $750-999 g$ & $50(29.6 \%)$ & $43(27.7 \%)$ & \\
\hline $1000-1249 g$ & $34(20.1 \%)$ & $36(23.2 \%)$ & \\
\hline$\geq 1250 \mathrm{~g}$ & $56(33.1 \%)$ & $54(34.8 \%)$ & \\
\hline Male sex & $94(55.6 \%)$ & $84(54.2 \%)$ & 0.80 \\
\hline Antenatal corticosteroids & & & 0.82 \\
\hline None & $8(4.7 \%)$ & $9(5.8 \%)$ & \\
\hline Not completed & $53(31.4 \%)$ & $43(27.7 \%)$ & \\
\hline Completed & $108(63.9 \%)$ & $103(66.5 \%)$ & \\
\hline \multicolumn{4}{|c|}{ GA (weeks+days) per category of corticosteroids, mean (SD) } \\
\hline None & $27+3(13)$ & $28+0(10)$ & 0.44 \\
\hline Not completed & $28+2(14)$ & $28+5(15)$ & 0.21 \\
\hline Completed & $27+6(15)$ & $27+6(14)$ & 0.83 \\
\hline \multicolumn{4}{|l|}{ Apgar, median (IQR) } \\
\hline $1 \mathrm{~min}$ & $6(4.0-6.5)$ & $5(4-7)$ & 0.89 \\
\hline $5 \mathrm{~min}$ & $7(6-8)$ & $7(6-8)$ & 0.70 \\
\hline Umbilical cord pH, median (IQR) & $7.30(7.26-7.34)$ & $7.30(7.24-7.34)$ & 0.48 \\
\hline Maximal $\mathrm{FiO}_{2}$ before $\mathrm{SF}$, median (IQR) & $35(30-45)$ & $40(33.75-50)$ & 0.15 \\
\hline Time birth-SF (hours), median (IQR) & $2(1.5-3.75)$ & $2.5(2-5)$ & $0.001^{*}$ \\
\hline
\end{tabular}

GA Gestational age, LISA Less Invasive Surfactant Administration, GA Gestational age

The study groups were comparable with regard to gestational age, birth weight, sex, Apgar score, umbilical cord $\mathrm{pH}$, maximal $\mathrm{FiO}_{2}$ before SF therapy and administration of antenatal steroids. Gestational age was also compared per category of antenatal steroids. This analysis showed no significant differences as well.

\section{Diagnostic procedures}

Diagnostic features of the study population are shown in Table 2. Patients treated with LISA underwent fewer Xrays compared to controls (median 1 vs. $3, p<0.001$ ), especially with regard to chest X-rays (median 1.0 vs. 2.0, $p<0.001$ ). Besides, the absolute number of collected blood gas samples was reduced in the first week of life,

Table 2 Diagnostic features of LISA group compared to control group

\begin{tabular}{llll}
\hline & LISA $(\boldsymbol{n}=169)$ & Control $(\boldsymbol{n}=155)$ & $\boldsymbol{p}$-value \\
\hline $\begin{array}{l}\text { X-rays, median (IQR) } \\
\text { Chest 1st week }\end{array}$ & $1.0(0-2)$ & $2.0(1-4)$ & $<.0(1-4)$ \\
All 1st week & $1.0(0-3)$ & & $<0.001^{*}$ \\
Laboratory tests in 1st week, median (IQR) & & $5.0(3-11)$ & $<0.001^{*}$ \\
Blood gas & $3.0(1-6)$ & $5.0(4-7)$ & 0.108 \\
Haematology & $6.0(4-8)$ & $8.0(7-10)$ & 0.481 \\
$\quad$ Chemistry & $8.0(7-9)$ & $120(77.9 \%)$ & $0.046^{*}$ \\
Blood culture for suspected EOS & $115(68.0 \%)$ & &
\end{tabular}

*shows significance $(p<0.05)$ 
Table 3 Treatment features of LISA group compared to control group in the first week of life

\begin{tabular}{|c|c|c|c|}
\hline & $\operatorname{LISA}(\boldsymbol{n}=169)$ & Control $(\boldsymbol{n}=155)$ & $\boldsymbol{p}$-value \\
\hline \multicolumn{4}{|l|}{ Surfactant } \\
\hline Multiple gifts & $55(32.5 \%)$ & $33(21.3 \%)$ & $0.023^{*}$ \\
\hline$\geq 1$ endotracheal tube gift & $35(20.7 \%)$ & 155 (100\%) & $<0.001^{*}$ \\
\hline Initial dose (mg/kg), median (IQR) & $189(162-207)$ & $148(126-174)$ & $<0.001^{*}$ \\
\hline Total dose (mg/kg), median (IQR) & $208(178-329)$ & 160 (129-198) & $<0.001^{*}$ \\
\hline \multicolumn{4}{|l|}{ Respiratory support } \\
\hline Intubation & 65 (38.5\%) & 155 (100\%) & $<0.001^{*}$ \\
\hline MV $12 \mathrm{~h}$ & $60(35.5 \%)$ & $119(76.8 \%)$ & $<0.001^{*}$ \\
\hline MV (days), median (IQR) & $0.0(0-1)$ & $1.0(1-3)$ & $<0.001^{*}$ \\
\hline \multicolumn{4}{|l|}{ Drug prescriptions } \\
\hline Morphine & $20(11.8 \%)$ & $23(14.8 \%)$ & 0.426 \\
\hline Midazolam & $3(1.8 \%)$ & $1(0.6 \%)$ & 0.358 \\
\hline Inotropes & $16(9.5 \%)$ & $28(18.1 \%)$ & $0.024^{*}$ \\
\hline Insulin & $8(4.7 \%)$ & $13(8.4 \%)$ & 0.182 \\
\hline Antibiotics for suspected EOS (days), median (IQR) & $3(2-6)$ & $5(3-7)$ & $<0.001^{*}$ \\
\hline PDA treatment courses & & & 0.658 \\
\hline None & $91(53.8 \%)$ & $84(54.2 \%)$ & \\
\hline 1 & $46(27.2 \%)$ & $35(22.6 \%)$ & \\
\hline$>1$ & $32(18.9 \%)$ & $36(23.2 \%)$ & \\
\hline Erythrocyte transfusions & & & $0.003^{*}$ \\
\hline None & $127(75.1 \%)$ & $90(58.1 \%)$ & \\
\hline 1 & $23(13.6 \%)$ & $37(23.9 \%)$ & \\
\hline$>1$ & $19(11.2 \%)$ & $28(18.1 \%)$ & \\
\hline Thrombocyte transfusions & & & 0.458 \\
\hline None & $153(90.5 \%)$ & $136(87.7 \%)$ & \\
\hline 1 & $10(5.9 \%)$ & $12(7.7 \%)$ & \\
\hline$>1$ & $6(3.6 \%)$ & $7(4.5 \%)$ & \\
\hline \multicolumn{4}{|l|}{ Enteral feeding (mL/kg), median (IQR) } \\
\hline Day 5 & $88(50-120)$ & $60(40-120)$ & 0.051 \\
\hline Day 7 & $120(80-160)$ & $100(60-140)$ & $0.048^{*}$ \\
\hline Umbilical catheter & $64(37.9 \%)$ & $80(51.6 \%)$ & $0.013^{*}$ \\
\hline Phototherapy (days), mean (SD) & $2.1(1.8)$ & $2.1(1.6)$ & 0.826 \\
\hline
\end{tabular}

*shows significance $(p<0.05)$

MV $12 \mathrm{~h}$ Mechanical ventilation for more than $12 \mathrm{~h}$, PDA Patent ductus arteriosus

showing almost twice as much collections in the controls (median 3 vs. $5, p<0.001$ ). In the LISA group, $68.0 \%$ of patients were treated for suspected EOS, compared to $77.9 \%$ in control group $(p=0.046)$. No differences between the study groups were found in the number of other blood samples for laboratory in the first week.

\section{Treatment}

Treatment features for both groups are shown in Table 3. Patients treated with LISA needed more than one SF dose in $32.5 \%$ of cases, compared to $21.3 \%$ in the control group $(p=0.023)$. In the LISA treated infants, $20.7 \%$ received a second dose of SF via an endotracheal tube after intubation. Initial and total dose of SF were higher in LISA group and showed a difference between the study groups of 41 and $48 \mathrm{mg} / \mathrm{kg}$, respectively ( $p<$ 0.001). In infants treated with LISA, 38.5 and $35.5 \%$ of patients were intubated in the first week and needed mechanical ventilation for at least $12 \mathrm{~h}$, respectively. The latter was needed in $78.8 \%$ of patients in the control group $(p<0.001)$. Control patients were treated with inotropic drugs roughly twice as much, compared to LISA patients $(18.1 \%$ vs. $9.5 \%, p=0.024)$. Duration of empirical antibiotic treatment for suspected (blood 
Table 4 Complications of LISA group compared to control group

\begin{tabular}{llll}
\hline & LISA $(\boldsymbol{n}=169)$ & Control $(\boldsymbol{n}=155)$ & $\boldsymbol{p}$-value \\
\hline Supplementary oxygen at day 28 in survivors & $79(49.7 \%)$ & $63(45.3 \%)$ & 0.452 \\
Surgical ligation of PDA & $9(5.3 \%)$ & $15(9.7 \%)$ & 0.135 \\
Abdominal surgery & $9(5.3 \%)$ & $7(4.5 \%)$ & 0.737 \\
IVH grade 3-4 & $11(6.5 \%)$ & $13(8.4 \%)$ & 0.519 \\
ROP laser surgery & $6(3.6 \%)$ & $4(2.6 \%)$ & 0.614 \\
Pneumothorax & $3(1.8 \%)$ & $6(3.9 \%)$ & 0.320 \\
Mortality during NICU admission & $14(8.3 \%)$ & $16(10.3 \%)$ & 0.527 \\
\hline
\end{tabular}

*shows significance $(p<0.05)$

$P D A$ Patent ductus arteriosus, IVH Intraventricular hemorrhage, ROP Retinopathy of prematurity

culture negative) EOS was longer in control patients (median 5.0 vs. 3.0 days, $p<0.001$ ). Erythrocyte transfusions were administered more frequently in the control group compared to LISA group $(41.9 \%$ vs. $24.9 \%, p=$ 0.001). The increase of enteral feeding was faster in the LISA group compared to controls, with a larger volume on day seven ( 120 vs. $100 \mathrm{~mL} / \mathrm{kg}, p=0.048)$. In the first week of life, LISA patients received an umbilical catheter in $37.9 \%$ of the cases, compared to $51.6 \%$ of control patients $(p=0.013)$. No differences between the groups were found considering PDA treatment courses, thrombocyte transfusions or days on phototherapy in the first week of life.

\section{Complications of prematurity}

As shown in Table 4, no statistically significant differences were found in complications of prematurity.

\section{Discussion}

In this study, we investigated the effects of LISA introduction in a tertiary NICU on several diagnostic and therapeutic procedures in the first week of life. We found that LISA implementation was associated with remarkable changes in neonatal practice. To the best of our knowledge this study is the first that provides insight into changes in NICU practice following LISA implementation, compared to classical SF administration. Previous studies in this field have only focused on variables related to respiratory support, pulmonary outcomes and complications $[3,4,11]$.

Both groups were similar regarding baseline characteristics. Postmenstrual age birthweight and $\mathrm{FiO} 2$ before SF treatment not significantly different between groups. The threshold for surfactant treatment was identical in both groups. Therefore the groups comparable to each other.

With respect to diagnostic procedures, LISA was associated with fewer X-rays. This is desirable, since all ionizing irradiation will increase the cumulative risk for radiation induced tumorigenesis [12]. This finding might be explained because X-rays were mostly performed to verify endotracheal tube position before administering
SF. We did not routinely perform X-rays to establish diagnosis of RDS, but relied on the clinical presentation. However, when diagnosis of RDS was questioned, or when pneumothorax or atelectasis was suspected, a chest X-ray was performed as well.

In LISA patients, fewer blood gas analyses and collections were performed. We have used throughout the entire study period we have used volume-targeted ventilation and performed non-invasive carbon dioxide monitoring (transcutaneous and/or end-tidal) in our NICU centre. Fewer blood gas sampling leads to a reduction in blood loss and skin breaking procedures. We expect that this difference in blood gas sampling will even be larger in centres who do not use volumetargeted ventilation and non-invasive carbon dioxide monitoring [13-15]. The saved resources on blood-gas measurements are a direct pharmaco-economic saving. Unfortunately, we have no numbers to quantify this saving in the present study.

Despite the higher initial dose of administered SF in LISA group, those patients needed redosing more often. Both the higher initial dose and redosing more frequently might be due to the knowledge clinicians gained on SF spill, loss of surfactant in de application device and a different pulmonary distribution of SF, which are reported to contribute to a higher required dose of SF for LISA patients $[16,17]$. In addition, a SF dose of fewer than $200 \mathrm{mg} / \mathrm{kg}$ is associated with LISA failure. This difference therefore possibly contributes to the higher rate of multiple administered gifts of SF in LISA group, since other risk factors for LISA failure (such as male gender) were comparable between the study groups [18]. Regarding respiratory support, reduction in the need for mechanical ventilation when using LISA is a well-established finding, which is reconfirmed in our results [6]. However, we did not find a significant difference in short term respiratory outcome.

An interesting and important finding is the lower incidence and shorter duration of antibiotic treatment for suspected EOS in patients treated with LISA. The Dutch protocol on EOS, which was based on the NICE 
guideline, mandates a critical review before the start and continuation of antibiotic treatment after $48 \mathrm{~h}$ of therapy. This protocol did not change during the study period. There was neither a significant difference in culture-proven EOS between LISA patients and controls (3.0\% vs $1.3 \%, p=0.302)$.

The adverse effects of antibiotics were already known before the study period. Antibiotic use in early life is known to disturb microbiota development in preterm infants [19]. Besides, reduction of unnecessary antibiotic treatment is of importance, as each day of prolonged early use is associated with an increased risk for lateonset sepsis (LOS), NEC or death [20]. However, reducing antibiotic use in daily NICU practice is still challenging. The decision to start and stop antibiotic therapy for suspected EOS is partly based on the assessment of the attending physician of the clinical condition of the infant and therefore subjective [11]. A preterm infant on non-invasive respiratory support is likely to be considered in clinically better condition than a mechanical ventilated infant. Therefore, the introduction of LISA, leaving less infants on mechanical ventilation, may have helped in deciding not to start or early discontinue antibiotic treatment for suspected EOS.

A large difference was found in the need for inotropic support between both study groups, with a significant lower need in LISA patients. This may be a logical finding, as sedatives and analgesics, which are both frequently used with endotracheal intubation and mechanical ventilation, often induce hypotension as side effect [21-23]. Moreover, mechanical ventilation itself might more often lead to hypotension as well [24]. During the study period no delayed cord clamping was performed in our NICU centre, which therefore did not affect our observations. The early use of inotropes is associated with severe IVH and increased mortality [25]. Therefore, we hypothesise that reduction in the use of inotropes may explain the lower rate of severe IVH in LISA treated infants in the NINSAPP study [26]. Furthermore, LISA was associated with a decrease of erythrocyte transfusions. The most logical explanation is the use of lower thresholds for erythrocyte transfusion in ventilated infants compared to non-ventilated infants in the Dutch protocol for blood transfusions [27]. Studies suggest that early erythrocyte transfusions are associated with an increased risk of severe IVH, ROP and mortality [28-30]. Therefore, the LISA associated reduction of may be of benefit for the patient.

Based on our findings, implementation of LISA may help in advancing enteral feeding, which resulted in higher amounts of enteral feeding at day 7 in LISA patients. According to our local feeding protocol, which did not change between the two study periods, preterm infants $<27$ weeks of gestation or infants with birth weight $<3$ rd percentile received trophic feeding for 2 to 3 days. Hereafter, feeding was increased with $20 \mathrm{~mL} / \mathrm{kg} /$ day if well tolerated (guided by clinical appearance, abdominal distension and/or tenderness, emesis, and large gastric residuals). The remaining infants started with enteral feeding immediately. Enteral feeding might be tolerated better by non-ventilated infants and may be explained partly by the lower but not significant difference in morphine administration. Moreover, the attending clinician could be less inclined to withhold feeding advancement in non-ventilated infants, who are deemed in a better clinical condition more often. Aggressive feeding strategies may reduce the risk of late-onset sepsis, without increasing the risk of NEC [31, 32]. Therefore, we think that this difference in enteral feeding advancement may be beneficial for those infants. We hypothesize that this finding may have contributed to the observation that $55.9 \%$ of infants in LISA group, compared to $32.0 \%$ in control group, having regained birth weight in the first week of life $(p<0.001)$.

In this study, we did not find statistically significant differences in complications of prematurity between our groups. We think this is due to our small study groups. It would be interesting to have data on our primary outcomes from studies in which those differences in complications were found. Unfortunately, we could not provide data on BPD incidence and severity, as a large proportion infants were transferred to other hospitals before the postmenstrual age of 36 weeks and no oxygen reduction test was performed. Therefore we were restricted to report only on supplementary oxygen at 28 days as proxy diagnosis of developing BPD.

Overall, we found several changes in NICU practices in early life, which may have beneficial effects on shortterm and long-term outcomes of preterm infants with RDS treated with LISA and lower use of healthcare resources. In this study, we did not focus on the long term outcome, as groups were considered too small to find statistically significant effects. We think this is the reason we found no difference in short-term outcomes as well. However, in future meta-analyses of studies with LISA including large groups of infants, effects of changes in NICU care aspects on these outcomes may be of interest. Besides, this study was performed on a single room maternity care with a special interest in family centred care. We consider a reduction in skin breaking procedures and fewer diagnostic procedures, which lead to less radiation load, as beneficial for these infants. Moreover, the presence of umbilical catheters, inotropic medication and respiratory instability are often barriers for Kangaroo care. The significant reduction in these interventions, will make it easier to start with Kangaroo care early in life, which, in our view, is of importance for these infants and their parents. Regrettably, we did not 
have objective data about the amount and duration of Kangaroo care in the first week of life. At last, the reduction in diagnostic and therapeutic procedures may reduce costs, although this may not weigh up against the costs of higher amount of administered SF in the LISA patients. However, this should be investigated in an extensive cost-effectiveness study.

A main strength of this study is the use of strict local protocols in our NICU, which reduces the variation of care among the attending neonatologist and nurses. Our protocols regarding enteral feeding, EOS or blood transfusions did not change during the study period. Another strength is that data was collected from electronic patient files, providing complete and reliable data.

However, there are several limitations that need to be acknowledged. Most importantly, this is a historical cohort study, with evident risks of confounding. Although protocols on the studied parameters did not change, implicit changes in NICU care, regardless of LISA implementation may have taken place which confounded results. However, baseline characteristics (gestational age, birth weight, sex, antenatal steroids, Apgar score, umbilical cord $\mathrm{pH}$ ) and RDS severity (determined as $\mathrm{FiO}_{2}$ before surfactant) were similar between both study groups. Therefore, in our opinion, the observed significant differences in diagnostic and treatment procedures are largely explained by the introduction of LISA. However, in order to confirm our observations, the changes in NICU care associated with LISA should may be subject of investigation in future randomized trials or a prospective cost-effectiveness study. Furthermore, our findings may not be extrapolated easily to other NICU centres implementing LISA, as this was a single centre study in the Dutch healthcare setting. However, our study provides items that other centres may evaluate before and after the introduction of LISA in order to assess the effects on care, outcome and resource allocation.

\section{Conclusion}

In conclusion, our findings demonstrate that introduction of LISA, as one of the minimally invasive surfactant procedures, has contributed to a change in practice towards fewer diagnostic and therapeutic interventions in preterm infants with RDS. We hypothesize that the reduction in diagnostic and therapeutic interventions in these vulnerable infants may explain the fewer complications after LISA in larger study groups and provides more opportunities for Kangaroo care. In addition, the allocation of healthcare resources changed, with more surfactant being administered with LISA but less $\mathrm{x}$-rays and blood gas analyses being performed. These findings emphasize the beneficial effects of using LISA as the primary management of RDS with exogenous SF requirement.

\section{Abbreviations}

CPAP: Continuous positive airway pressure; GA: Gestational age; EOS: Early Onset Sepsis; IQR: Interquartile range; IVH: Intraventricular haemorrhage; LISA: Less invasive surfactant administration; NEC: Necrotizing enterocolitis; NICU: Neonatal intensive care unit; PDA: Patent ductus arteriosus; RDS: Respiratory distress syndrome; ROP: Retinopathy of prematurity; SD: Standard deviation; SF: Surfactant

\section{Acknowledgements}

Non applicable.

\section{Authors' contributions}

IB: study design, data acquisition and analysis, interpretation of data, drafted manuscript, revised manuscript. LJ: data acquisition, data interpretation, revised manuscript. JD: data analysis, interpretation of data. BK: study design, interpretation of data, substantially revised manuscript. PA: study design, interpretation of data, substantially revised manuscript. HN: conceptualised study, study design, data acquisition and analysis, interpretation of data, substantially revised manuscript. All authors have read and approved the final manuscript.

\section{Funding}

Not applicable.

\section{Availability of data and materials}

The datasets used and/or analysed during the current study are available from the corresponding author on reasonable request.

\section{Ethics approval and consent to participate}

The medical ethics committee of the Máxima Medical Centre Veldhoven, the Netherlands approved this protocol (under number N18.062).

\section{Consent for publication \\ Not applicable.}

\section{Competing interests}

The authors declare that they have no competing interests.

\section{Author details}

'Department of Neonatology, Máxima Medical Centre, De Run 4600, 5504 DB Veldhoven, The Netherlands. 'ª́xima Medical Centre Academy, Máxima Medical Centre, Veldhoven, the Netherlands. ${ }^{3}$ Department of Pediatrics, Maastricht University Medical Centre, P Debyelaan 20, 6229 HX Maastricht, The Netherlands.

Received: 3 April 2020 Accepted: 27 August 2020

Published online: 03 September 2020

References

1. Dargaville PA, Gerber A, Johansson S, De Paoli AG, Kamlin CO, Orsini F, et al. Incidence and Outcome of CPAP Failure in preterm infants. Pediatrics. 2016; 138(1):e20153985.

2. Brix N, Sellmer A, Jensen MS, Pedersen LV, Henriksen TB. Predictors for an unsuccessful INtubation-SURfactant-Extubation procedure: a cohort study. BMC Pediatr. 2014;14:155.

3. Vento G, Pastorino R, Boni L, Cota F, Carnielli V, Cools F, et al. Efficacy of a new technique - INtubate-RECruit-SURfactant-Extubate - "IN-REC-SUR-E" - in preterm neonates with respiratory distress syndrome: study protocol for a randomized controlled trial. Trials. 2016:17:414.

4. Barkhuff WD, Soll RF. Novel surfactant administration techniques: will they change outcome? Neonatology. 2019;115(4):411-22.

5. Gopel W, Kribs A, Ziegler A, Laux R, Hoehn T, Wieg C, et al. Avoidance of mechanical ventilation by surfactant treatment of spontaneously breathing preterm infants (AMV): an open-label, randomised, controlled trial. Lancet. 2011;378(9803):1627-34.

6. Aldana-Aguirre JC, Pinto M, Featherstone RM, Kumar M. Less invasive surfactant administration versus intubation for surfactant delivery in preterm infants with respiratory distress syndrome: a systematic review and metaanalysis. Arch Dis Child Fetal Neonatal Ed. 2017;102(1):F17-f23.

7. Hartel C, Paul P, Hanke K, Humberg A, Kribs A, Mehler K, et al. Less invasive surfactant administration and complications of preterm birth. Sci Rep. 2018;8(1):8333. 
8. Klebermass-Schrehof K, Wald M, Schwindt J, Grill A, Prusa AR, Haiden N, et al. Less invasive surfactant administration in extremely preterm infants: impact on mortality and morbidity. Neonatology. 2013;103(4):252-8.

9. Lau CSM, Chamberlain RS, Sun S. Less invasive surfactant administration reduces the need for mechanical ventilation in preterm infants: a metaanalysis. Glob Pediatr Health. 2017:4:2333794x17696683.

10. Sweet DG, Carnielli V, Greisen G, Hallman M, Ozek E, Te Pas A, et al. European consensus guidelines on the Management of Respiratory Distress Syndrome - 2019 update. Neonatology. 2019;115(4):432-50.

11. Klingenberg C, Kornelisse RF, Buonocore G, Maier RF, Stocker M. Culturenegative early-onset neonatal Sepsis - at the crossroad between efficient Sepsis care and antimicrobial stewardship. Front Pediatr. 2018;6:285.

12. Kleinerman RA. Cancer risks following diagnostic and therapeutic radiation exposure in children. Pediatr Radiol. 2006;36(Suppl 2):121-5.

13. Klingenberg C, Wheeler KI, McCallion N, Morley CJ, Davis PG. Volumetargeted versus pressure-limited ventilation in neonates. Cochrane Database Syst Rev. 2017;10:Cd003666.

14. Kugelman A, Golan A, Riskin A, Shoris I, Ronen M, Qumqam N, et al. Impact of continuous capnography in ventilated neonates: a randomized, multicenter study. J Pediatr. 2016;168:56-61.e2.

15. Mukhopadhyay S, Maurer R, Puopolo KM. Neonatal transcutaneous carbon dioxide monitoring--effect on clinical management and outcomes. Respir Care. 2016;61(1):90-7.

16. Niemarkt HJ, Kuypers E, Jellema R, Ophelders D, Hutten M, Nikiforou M, et al. Effects of less-invasive surfactant administration on oxygenation, pulmonary surfactant distribution, and lung compliance in spontaneously breathing preterm lambs. Pediatr Res. 2014;76(2):166-70.

17. De Luca D, Minucci A, Gentile L, Capoluongo ED. Surfactant inadvertent loss using feeding catheters or endotracheal tubes. Am J Perinatol. 2014;31(3):209-12.

18. Janssen LC, Van Der Spil J, van Kaam AH, Dieleman JP, Andriessen P, Onland W, et al. Minimally invasive surfactant therapy failure: risk factors and outcome. Arch Dis Child Fetal Neonatal Ed. 2019;104(6):F636-f42.

19. Schulfer A, Blaser MJ. Risks of antibiotic exposures early in life on the developing microbiome. PLoS Pathog. 2015;11(7):e1004903.

20. Ting JY, Roberts A, Sherlock R, Ojah C, Cieslak Z, Dunn M, et al. Duration of initial empirical antibiotic therapy and outcomes in very low birth weight infants. Pediatrics. 2019;143(3):e20182286.

21. Anand KJ, Hall RW, Desai N, Shephard B, Bergqvist LL, Young TE, et al. Effects of morphine analgesia in ventilated preterm neonates: primary outcomes from the NEOPAIN randomised trial. Lancet. 2004;363(9422):1673-82

22. Welzing L, Kribs A, Eifinger F, Huenseler C, Oberthuer A, Roth B. Propofol as an induction agent for endotracheal intubation can cause significant arterial hypotension in preterm neonates. Paediatr Anaesth. 2010;20(7):605-11.

23. Simons $\mathrm{SH}$, van der Lee $\mathrm{R}$, Reiss IK, van Weissenbruch MM. Clinical evaluation of propofol as sedative for endotracheal intubation in neonates. Acta Paediatr. 2013;102(11):e487-92

24. Lakkundi A, Wright I, de Waal K. Transitional hemodynamics in preterm infants with a respiratory management strategy directed at avoidance of mechanical ventilation. Early Hum Dev. 2014;90(8):409-12.

25. St Peter D, Gandy C, Hoffman SB. Hypotension and adverse outcomes in prematurity: comparing definitions. Neonatology. 2017;111(3):228-33.

26. Kribs A, Roll C, Gopel W, Wieg C, Groneck P, Laux R, et al. Nonintubated surfactant application vs conventional therapy in extremely preterm infants: a randomized clinical trial. JAMA Pediatr. 2015;169(8):723-30.

27. von Lindern J, Brand A, Lopriore E. Revisie van de richtlijn 'bloedtransfusie'. Ned Tijdschr Geneeskd. 2012;156:A4795.

28. Wang YC, Chan OW, Chiang MC, Yang PH, Chu SM, Hsu JF, et al. Red blood cell transfusion and clinical outcomes in extremely low birth weight preterm infants. Pediatr Neonatol. 2017:58(3):216-22.

29. Baer VL, Lambert DK, Henry E, Snow GL, Christensen RD. Red blood cell transfusion of preterm neonates with a grade 1 intraventricular hemorrhage is associated with extension to a grade 3 or 4 hemorrhage. Transfusion. 2011;51(9):1933-9.

30. Christensen RD, Baer VL, Lambert DK, Ilstrup SJ, Eggert LD, Henry E. Association, among very-low-birthweight neonates, between red blood cell transfusions in the week after birth and severe intraventricular hemorrhage. Transfusion. 2014;54(1):104-8

31. Oddie SJ, Young L, McGuire W. Slow advancement of enteral feed volumes to prevent necrotising enterocolitis in very low birth weight infants. Cochrane Database Syst Rev. 2017:8:Cd001241.
32. El Manouni El Hassani S, Berkhout DJC, Niemarkt HJ, Mann S, de Boode WP, Cossey $\mathrm{V}$, et al. Risk factors for late-onset Sepsis in preterm infants: a multicenter case-control study. Neonatology. 2019;116(1):42-51.

\section{Publisher's Note}

Springer Nature remains neutral with regard to jurisdictional claims in published maps and institutional affiliations.
Ready to submit your research? Choose BMC and benefit from:

- fast, convenient online submission

- thorough peer review by experienced researchers in your field

- rapid publication on acceptance

- support for research data, including large and complex data types

- gold Open Access which fosters wider collaboration and increased citations

- maximum visibility for your research: over $100 \mathrm{M}$ website views per year

At BMC, research is always in progress.

Learn more biomedcentral.com/submissions 